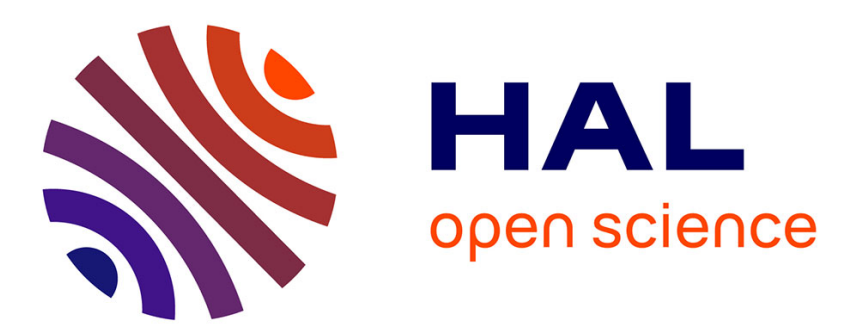

\title{
Pixel super-resolution in digital holography by regularized reconstruction
}

Corinne Fournier, Frédéric Jolivet, Loïc Denis, Nicolas Verrier, Eric Thiebaut, Cédric Allier, T Fournel

\section{- To cite this version:}

Corinne Fournier, Frédéric Jolivet, Loïc Denis, Nicolas Verrier, Eric Thiebaut, et al.. Pixel superresolution in digital holography by regularized reconstruction. Applied optics, 2017, 56 (1), pp.69 77. 10.1364/AO.56.000069 • ujm-01575914

\section{HAL Id: ujm-01575914}

\section{https://hal-ujm.archives-ouvertes.fr/ujm-01575914}

Submitted on 21 Aug 2017

HAL is a multi-disciplinary open access archive for the deposit and dissemination of scientific research documents, whether they are published or not. The documents may come from teaching and research institutions in France or abroad, or from public or private research centers.
L'archive ouverte pluridisciplinaire HAL, est destinée au dépôt et à la diffusion de documents scientifiques de niveau recherche, publiés ou non, émanant des établissements d'enseignement et de recherche français ou étrangers, des laboratoires publics ou privés. 


\title{
Pixel Super-Resolution in digital holography by regularized reconstruction.
}

\author{
C. Fournier ${ }^{1, *}$, F. Jolivet ${ }^{1}$, L. Denis ${ }^{1}$, N. Verrier ${ }^{2}$, E. Thiebaut ${ }^{3,4}$, C. Allier ${ }^{5}$, And T. \\ FOURNEL ${ }^{1}$ \\ ${ }^{1}$ Laboratoire Hubert Curien - UMR 5516-CNRS-Université Jean Monnet- 18 Rue du Professeur Benoît Lauras F-42000 Saint-Etienne, France \\ ${ }^{2}$ Laboratoire Modélisation, Intelligence, Processus, Systèmes-EA2332, IUT Mulhouse, 61 rue A. Camus, 68093 Mulhouse CEDEX, France \\ ${ }^{3}$ Univ. Lyon, Univ. Lyon 1, ENS de Lyon, CNRS, Centre de Recherche Astrophysique de Lyon UMR5574, F-69230, Saint-Genis-Laval, France \\ ${ }^{4}$ CNRS, UMR 5574, Ecole Normale Supérieure de Lyon, 69007 Lyon, France \\ ${ }^{5}$ CEA, LETI, MINATEC, 17 rue des Martyrs, 38054 Grenoble cedex 9, France \\ *Corresponding author: corinne.fournier@univ-st-etienne.fr
}

Compiled November 22, 2016

\begin{abstract}
In-line digital holography (DH) and lensless microscopy are 3D imaging techniques used to reconstruct the volume of micro-objects in many fields. However, their performances are limited by the pixel size of the sensor. Recently, various pixel super-resolution algorithms for digital holography have been proposed. A hologram with improved resolution was produced from a stack of laterally shifted holograms, resulting in better resolved reconstruction than a single low-resolution hologram. Algorithms for superresolved reconstructions based on inverse problems approaches have already been shown to improve the 3D reconstruction of opaque spheres. Maximum a posteriori (MAP) approaches have also been shown capable of reconstructing the object field more accurately and more efficiently and to extend the usual field-of-view. Here we propose an inverse problem formulation for DH pixel super-resolution and an algorithm that alternates registration and reconstruction steps. The method is described in detail and used to reconstruct synthetic and experimental holograms of sparse 2D objects. We show that our approach improves both the shift estimation and reconstruction quality. Moreover, the reconstructed field-of-view can be expanded by up to a factor 3, thus making it possible to multiply the analyzed area 9 fold. () 2016 Optical Society of America
\end{abstract}

OCIS codes: (090.1995) Holography: Digital holography, (100.3190) Image processing: Inverse problems, (100.6640) Image processing: Superresolution; (100.3010) Image processing: Image reconstruction techniques.

http://dx.doi.org/10.1364/ao.XX.XXXXXX

\section{INTRODUCTION}

Since in-line holographic imaging was proposed by Dennis Gabor [1], followed by digital holographic (DH) imaging $[2,3]$, it has become a method of choice for a wide variety of applications including fluid mechanics, mechanical inspection, and biomedical imaging. The method, also known as lensless imaging [4], relies on a relatively inexpensive and easy to implement configuration in which no collection optics is needed to perform imaging. The role of the collection optics is advantageously replaced by data processing approaches aimed at simulating light back-propagation from the sensor plane to the plane where the objects to be investigated are located [5-7], thus enabling 3D imaging from the recording of one 2D hologram. Its cost-effectiveness associated with the democratization of high resolution, and high definition imaging sensors made it possible to develop on-chip wide field holographic microscopes [8] suited for the detection of bacteria and viruses [9, 10], cytometry [11], or the characterization of protein aggregates [12]. In this case, the resolution of the lensless microscope is driven by the pixel pitch of the chosen sensor, but can be enhanced using pixel super-resolution strategies [13].

Simulation of light back-propagation is still often used for hologram reconstruction because it is simple, although it is 
prone to border effects and twin image noise, which dramatically reduce the reconstruction signal to noise ratio (SNR) and consequently the accuracy of the reconstruction. Holographic reconstruction is also possible based on another paradigm. Instead of transforming the acquired hologram, the aim is to find the object transmittance that best matches the measurements. This inverse problem (IP) approach extracts more information from the hologram and has been shown to solve two major problems in digital holography: (i) the need to improve the accuracy of the reconstruction, (ii) the need to enlarge the field-of-view beyond the physical limit of the sensor [14-18]. It also leads to almost unsupervised algorithms (only a few tuning parameters are required). These approaches are sometimes referred to as compressive sensing methods [19-21].

To overcome the limitation caused by the pixel pitch of the hologram, in 2010, Ozcan's team introduced pixel superresolution (SR)[13], which was already used in other modalities [22,23]. These authors suggested it was possible to recover a higher resolution hologram using a stack of rigidly shifted lower resolution holograms and to reconstruct them by back-propagation. Recently, it was shown that coupling IP with pixel SR can improve the accuracy of hologram reconstruction even more [24, 25]. In this IP approach, a high resolution hologram is no longer computed, instead the reconstruction is performed directly from the hologram stack. However, despite their accuracy, these new DH-SR algorithms are based on a parametric reconstruction limited to the reconstruction of parametric objects (i.e. relying on a few parameters). For arbitrary shaped objects, the object 3D space can be sampled and a transmittance value estimated at each voxel. The image-hologram formation model can then be inverted using an a priori on the objects by means of a prior on the 3D object. This maximum posteriori (MAP) reconstruction has been shown to reduce twin images and to extend the usual field-of-view under sparse prior [17] or total variation prior [14, 18, 20, 26, 27].

We propose to combine pixel SR with IP approaches to perform optimal high resolution hologram reconstruction of arbitrary sparse objects from a stack of shifted holograms. This approach has already been used for conventional imaging [23], we apply it to DH. The specificity of DH makes the results unique: high accuracy registration, out-of-field reconstruction. In the first part of the article, the pixel super-resolution issue is presented from an IP point of view. Then, an algorithm that alternates registration steps and transmittance reconstruction is presented. Finally, the approach is validated with both synthetic and experimental data resulting in high SNR transmittance reconstruction over an enlarged field-of-view. Here the proof of concept is limited to the reconstruction of a $2 \mathrm{D}$ plane, but can be extended to 3D sparse objects.

\section{SR RECONSTRUCTION PROBLEM AS AN INVERSE PROBLEM}

Pixel SR algorithms in DH attempt to extract a high-resolution opacity distribution (real or complex valued) from a sequence of low-resolution holograms. This issue can be solved using an IP because the imaging model (direct model) is well-known. In this section, we first describe the direct model and then mathematically formalize the reconstruction problem.

\section{A. Direct model}

The direct model gives a mathematical expression of the hologram intensity as a function of the object opacity. Let us consider the simple case of sparse semi-opaque objects located in a plane and illuminated by a collimated coherent beam of wavelength $\lambda$. This object is described by its opacity distribution $\vartheta$, which is assumed to be real. A sensor positioned at a distance $z$ from the object plane (Fig. 1) records the intensity of the wave diffracted by the object.

Assuming the holographed object $l$ is narrow in width and satisfies $\pi l^{2} /(4 \lambda z) \ll 1$, the hologram intensity $I(x, y)$ can be approximated as an incoherent summation of diffraction patterns created by each point of the object plane characterized by its opacity $\vartheta$.

$$
I(x, y) \propto\left[1-2 . \vartheta * h_{z}\right](x, y)
$$

where $h_{z}$ stands for the real part of the free space propagation impulse response, $*$ for the 2D spatial convolution operator and $\alpha$ is the proportionality sign.

In the Fresnel diffraction regime [28] (i.e. $\left.z^{3} \gg \pi l^{4} /(64 \lambda)\right), h_{z}$ is given by:

$$
h_{z}(x, y)=\frac{1}{\lambda z} \sin \left(\frac{\pi\left(x^{2}+y^{2}\right)}{\lambda z}\right) \text {. }
$$

Accounting for pixel integration [29], a transversal shift of the sensor $\Delta^{i}=\left(\Delta x^{i}, \Delta y^{i}\right)$ and the sensor sampling, the intensity model of the $i^{\text {th }}$ hologram can also be expressed in a linear form:

$$
I^{i}\left(x_{p}, y_{p}\right) \propto\left[1-2 . \vartheta * h_{z} * \Pi * \delta_{\Delta^{i}}\right]\left(x_{p}, y_{p}\right)
$$




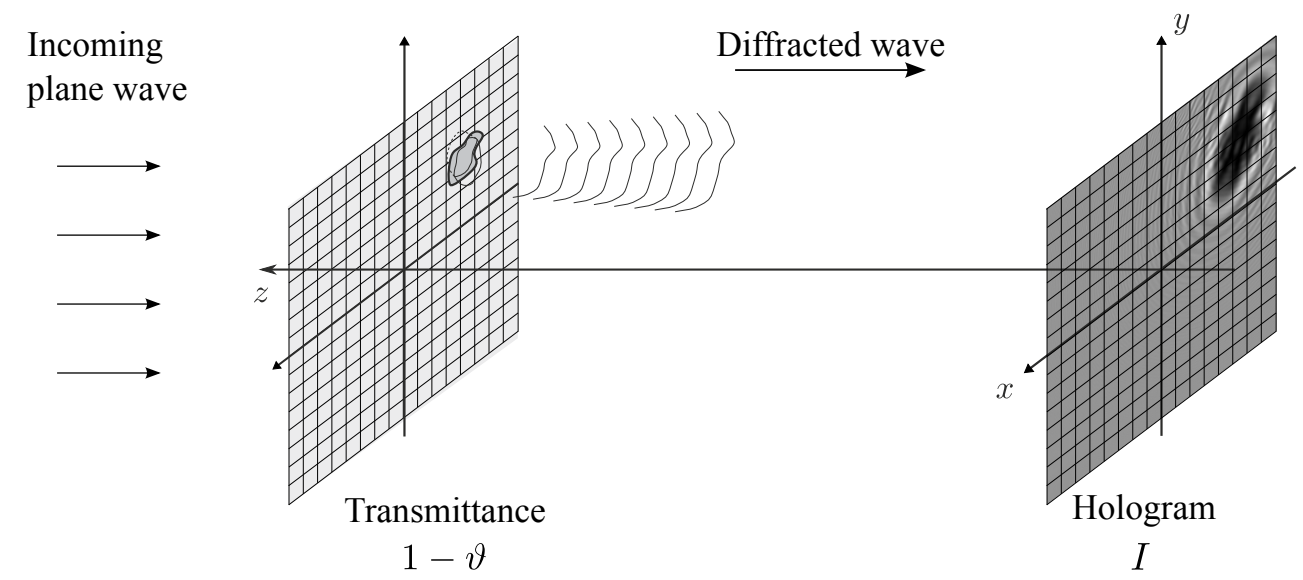

Fig. 1. Illustration of the in-line hologram formation model.

where $\left(x_{p}, y_{p}\right)$ is the center of the $p^{\text {th }}$ pixel, $\Pi$ is the 2D rectangular function representing the photo-sensitive area of a pixel, $\delta_{\Delta^{i}}$ is the Dirac distribution centered on $\left(\Delta x^{i}, \Delta y^{i}\right)$.

This expression can be rewritten as

$$
I^{i}\left(x_{p}, y_{p}\right) \propto\left[1-\vartheta * h_{z, \Delta^{i}}^{\Pi}\right]\left(x_{p}, y_{p}\right)
$$

with

$$
h_{z, \Delta^{i}}^{\Pi}=2 . h_{z} * \Pi * \delta_{\Delta^{i}} .
$$

In a pre-process step the holograms are divided by a background image (image with no object in the field), so that the proportionality factor is removed.

In the following, we use matrix notation as a simpler mathematical expression of the direct model. Bold notations are used to indicate a vector or a matrix. The image is represented in vector form by considering the $2 \mathrm{D}$ image as a $1 \mathrm{D}$ vector by stacking all columns of the image. The convolution of the kernel $h_{z, \Delta^{i}}^{\Pi}$ with the sampled opacity $\vartheta$ is thus expressed as the multiplication of a large discrete convolution matrix $\boldsymbol{H}_{\Delta^{i}}$ with the vector corresponding to the object opacity $\vartheta$. The matrix $\boldsymbol{H}_{\Delta^{i}}$ is made of pixel-shifted replications of $h_{z, \Delta^{i}}^{\Pi}$ put into a vector form.

The direct model $m^{i}$ representing the intensity of the $i^{\text {th }}$ hologram is in matrix notation:

$$
\boldsymbol{m}^{i}=\mathbf{1}-\boldsymbol{H}_{\Delta^{i} \vartheta}
$$

where 1 represents the offset and is a vector of $\mathrm{n}$ "ones", $\boldsymbol{H}_{\Delta^{i}}$ models diffraction and takes into account the relative displacement of the object with respect to the camera $\left(\Delta^{i}\right)$ and $\vartheta$ is the unknown opacity distribution of the object, common to all holograms.

Considering a centered model $\overline{\boldsymbol{m}}^{i}$ and a centered diffraction matrix $\overline{\boldsymbol{H}}$, the expression of the model is simplified [30] to:

$$
\overline{\boldsymbol{m}}^{i}=\overline{\boldsymbol{H}}_{\Delta^{i \vartheta}}
$$

with $\overline{\boldsymbol{m}}^{i}=\boldsymbol{m}^{i}-\frac{1}{n} \mathbf{1 1}^{t} \boldsymbol{m}^{i}$ and $\overline{\boldsymbol{H}}=-\boldsymbol{H}+\frac{1}{n} \mathbf{1 1}^{t} \boldsymbol{H}$.

From the point of view of implementation, to evaluate this model, the convolutions are computed in the Fourier space using Fast Fourier Transform. The sampling of the model is thus given by the opacity distribution sampling. A super-resolved opacity distribution leads to a super-resolved model:

$$
\overline{\mathbf{m}}^{i, S R}=\overline{\boldsymbol{H}}_{\Delta^{i}} \vartheta^{S R}
$$

\section{B. SR Reconstruction}

In an IP framework, the cost function enforces a certain fidelity of the final solution to the measured data. For the SR issue, the goal is to jointly reconstruct the super-resolved opacity from the whole hologram sequence. The fidelity term is thus composed of a sum of individual cost functions with a common super-resolved object opacity. Modeling the 
error between the modeled intensity and the measured hologram as white and Gaussian, the negative log-likelihood is proportional to:

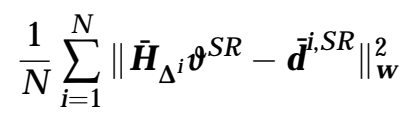

where:

- $N$ is the number of holograms,

- $\bar{d}^{i}$ is the $i^{\text {th }}$ centered hologram.

- the exponent $\left({ }^{S R}\right)$ indicates that data sampling is the same as opacity sampling $\vartheta^{S R}$; in practice zeros have been inserted in $\bar{d}^{i, S R}$ at the position of missing pixels (e.g. for a super-resolution factor of 2 , one column out of two are considered as missing (Fig. 3)),

- $w$ is the weight vector that accounts for missing data (for pixel that are missing the weight is 0 , otherwise it is 1 ). More generally, it can also account for non-uniform noise variance [15],[31],

- $\|\cdot\|_{w}^{2}$ is the weighted $L_{2}$ norm defined as: $\|\boldsymbol{u}\|_{w}^{2}=\langle\boldsymbol{u}, \boldsymbol{u}\rangle_{w}=\left(\sum_{p=1}^{n} w_{p} u_{p}^{2}\right) /\left(\sum_{p=1}^{n} w_{p}\right)$.

This inverse problem is ill-posed. In order to stabilize the inversion process, a regularization penalty function $\rho$, that enforces an a priori on the object to reconstruct, is added. This regularization is applied to the reconstructed superresolved object opacity $\vartheta^{S R}$.

The expression of the cost function becomes:

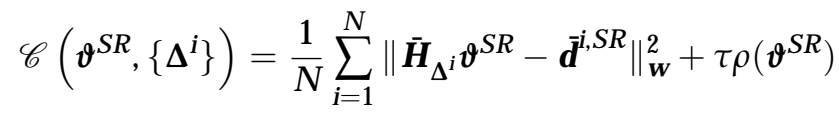

where the coefficient $\tau$ indicates the weight of the regularization term.

Reconstructing a super-resolved opacity using an IP framework is equivalent to solving the minimization problem:

$$
\hat{\vartheta}^{S R}=\underset{0 \leqslant \vartheta^{S R} \leqslant 1}{\arg \min }\left\{\min _{\Delta^{i}} \mathscr{C}\left(\vartheta^{S R},\left\{\Delta^{i}\right\}\right)\right\}
$$

where bound constraints are imposed on the opacity $\vartheta$ to account for the physics of absorption: the opacity of semiopaque objects being between 0 (transparent) and 1 (opaque).

This IP formulation provides a rigorous mathematical framework for the SR problem. Indeed, the shifted holograms of the sequence are modeled using the image formation model and consequently, no arbitrary interpolation function is used. The interpolation is implicitly made by the image formation model. Furthermore, the penalty function (based on an a priori on the objects) acts as a denoising step in the reconstruction. In the following section, we detail how the minimization in equation (11) is achieved.

\section{ALTERNATING OPTIMIZATION ALGORITHM}

The joint minimization of Eq. 11, consists in optimizing two different quantities $\vartheta^{S R}$ and $\left\{\boldsymbol{\Delta}^{i}\right\}_{i=1 . . N}$. It can be performed iteratively by alternating minimization [32]. The cost function is minimized with respect to the high resolution object opacity $\vartheta^{S R}$ and the shift parameters $\left\{\boldsymbol{\Delta}^{i}\right\}_{i=1 . . N}$ alternatively until convergence of the algorithm. Figure 2 represents the $k^{\text {th }}$ step of the alternating optimization. The two minimization steps are detailed below.

\section{A. Registration step}

It is widely accepted that the accuracy of the shift estimation in the image sequence is of paramount importance for the efficiency of SR algorithms [33]. The shifts are usually estimated relative to a reference image (e.g., the first one of the sequence). The estimation is then performed by minimizing the square difference between the first image and a shifted version of the $i^{\text {th }}$ image. As the accuracy of the estimate is subpixel, interpolation functions have to be used. Whatever the choice of the interpolation function (linear, cubic spline function, etc.), it is arbitrary. The use of the IP approach fixes this issue, as interpolation is implicitly performed by the image formation model. 


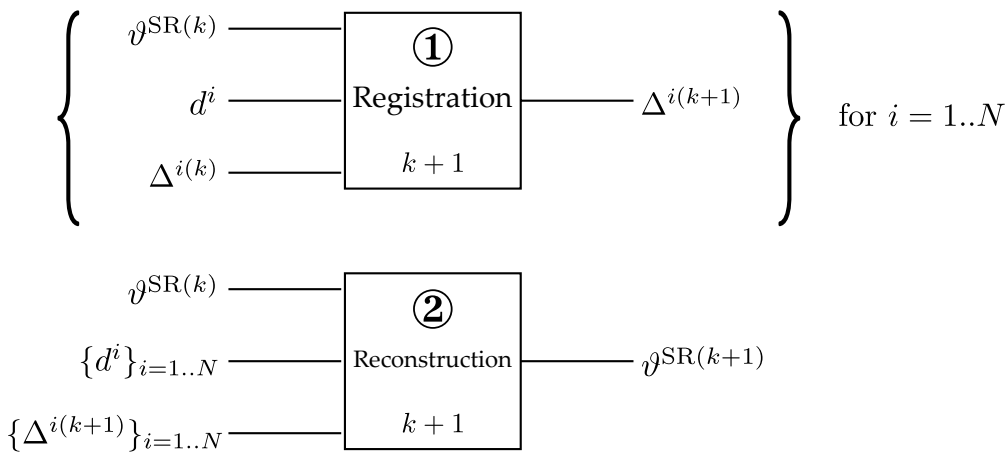

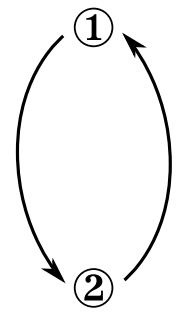

Alternate Optimization

Fig. 2. Illustration of the $k^{\text {th }}$ step of the alternating optimization algorithm.

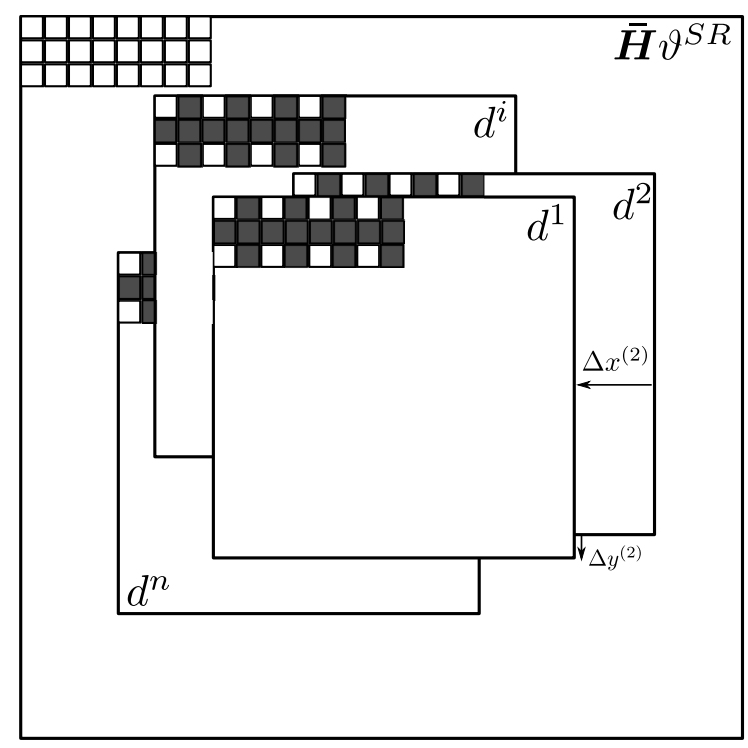

Fig. 3. Illustration of the shift estimation step for a SR factor of 2 . The unshifted and super-resolved hologram model $\left(\bar{H}_{\Delta^{i}} \vartheta^{S R}\right)$ is shown in the background. The shifted and low-resolution captured holograms $d^{i}$ are superimposed The gray pixels corresponds to missing data in the super-resolved space. The white pixels corresponds to the measured gray levels. The pixel size and the shift lengths are enlarged for the purpose of illustration. 
The transverse coordinate system $(O, x, y)$ of the first hologram is considered as reference. The opacity distribution is reconstructed relatively to this coordinate system. The shift $\Delta^{i}$ of the $i^{\text {th }}$ hologram relative to the first one is also the shift between the $i^{\text {th }}$ hologram and the image formation model $\bar{H} \vartheta^{S R}$. Thus, the problem is to minimize the residual between the shifted model and the $i^{\text {th }}$ hologram:

$$
\widehat{\Delta^{i}}=\underset{\Delta^{i}}{\arg \min }\left\|\overline{\boldsymbol{H}}_{\Delta^{i}} \vartheta^{S R}-\overline{\boldsymbol{d}}^{i, S R}\right\|_{w}^{2}
$$

An illustration is given in Fig. 3. The support of the model is larger than the data because of the diffraction and outof-field reconstruction of the opacity. Provided the opacity is well reconstructed, the shift estimate can be expected to be more accurate than by performing the registration on noisy holograms. Indeed, in our approach, the problem is equivalent to registering a large noiseless model with a hologram (which consists of a noisy shifted model), whereas in state-of-the-art approach, holograms are registered 2 by 2 . The noise variance in our registration step is theoretically divided by two. It theoretically decreases the standard error on the shifts by a factor $\sqrt{2}$ the standard error on the shifts. Furthermore there are no cropping effects, because the model is much larger than the holograms.

Direct minimization of this cost function is computationally too expensive because it involves the computation of a large model for each model evaluation (e.g. six times the width of the data if the SR factor is 2 and the field-of-view extension ratio is 3) and 6 FFT to compute the weighted centered correlation. In practice, the shift estimation can be performed in a sampled space of the shifts. Equation (12) can be rewritten as a maximization of the weighted normalized centered cross correlation [16]:

$$
\widehat{\Delta^{i}}=\underset{\Delta^{i}}{\arg \max } \frac{\left\langle\overline{\boldsymbol{H}}_{\Delta^{i}} \vartheta^{S R}, \overline{\boldsymbol{d}}^{i, S R}\right\rangle_{\boldsymbol{w}}}{\sqrt{\| \overline{\boldsymbol{H}}_{\Delta^{i} \vartheta^{S R} \|_{w}^{2}} \sqrt{\left\|\overline{\boldsymbol{d}}^{i, S R}\right\|_{w}^{2}}}}
$$

To avoid calculating multiple subpixel shifted models, the correlation is calculated on a map corresponding to integer shifts. The maximum of the correlation map is then positioned with subpixel accuracy by fitting parabolas (vertical and horizontal) on the correlation peak. The first guess concerning the shifts is obtained by correlation-based registration of the holograms.

\section{B. Opacity reconstruction step}

In the second optimization, the shifts are fixed and the opacity is estimated using a penalized maximum likelihood. Regularization may differ depending on the a priori on the object (e.g. total variation for objects with piecewise-constant opacity and sharp edges). Here, we assume a sparse semi-opaque object. A natural constraint is thus the $L_{1}$ norm of the opacity: $\rho\left(\vartheta^{S R}\right)=\left\|\vartheta^{S R}\right\|_{1}$. $\vartheta^{S R}$ is also assumed to be positive and less than 1 . The reconstructed opacity then corresponds to the solution of the following minimization problem:

$$
\widehat{\boldsymbol{\vartheta}}^{S R}=\underset{0 \leq \boldsymbol{\vartheta}^{S R} \leq 1}{\arg \min } \frac{1}{N} \sum_{i=1}^{N}\left\|\overline{\boldsymbol{H}}_{\boldsymbol{\Delta}^{i}} \boldsymbol{\vartheta}^{S R}-\overline{\boldsymbol{d}}^{i, S R}\right\|_{\boldsymbol{w}}^{2}+\tau\left\|\boldsymbol{\vartheta}^{S R}\right\|_{1}
$$

Under positivity constraints, the $L_{1}$ norm corresponds to the sum of the opacities: $\left\|\boldsymbol{\vartheta}^{S R}\right\|_{1}=\sum_{j} \vartheta_{j}^{S R}$.

The minimization problem (14) is a smooth optimization problem under bound constraints that can be solved efficiently using a limited-memory quasi-Newton method with bound constraints, such as VMLMB [34]. Proximal algorithms are also widely used for L1 minimization. Use of the FISTA algorithm [35] (an accelerated proximal method) to the problem (14) leads to Algorithm 1. We found that it converges slightly more slowly than VMLMB, but is useful because of its straightforward implementation, and could easily be accelerated by implementation on the GPU [36].

Algorithm 1: Reconstruction of a sparse distribution of opacities

input: holograms $\bar{d}^{i, S R}$ and shifts $\Delta^{i}$

output: reconstructed opacity distribution $\vartheta^{S R}$

initialization:

initial opacity distribution $\vartheta_{0}^{S R}$ (can be set to zero)

$\eta_{1} \leftarrow \vartheta_{0}^{S R}$

$t_{1} \leftarrow 1$ 
for $k=1,2, \ldots$

$$
\begin{aligned}
\boldsymbol{\vartheta}_{k}^{S R} & \leftarrow S_{\tau, 1}^{+}\left[\boldsymbol{\eta}_{k}+\frac{2}{N} \sum_{i=1}^{N} \overline{\boldsymbol{H}}_{\Delta^{i}}^{*} \cdot \operatorname{diag}(\boldsymbol{w}) \cdot\left(\overline{\boldsymbol{d}}^{i, S R}-\overline{\boldsymbol{H}}_{\Delta^{i}} \boldsymbol{\eta}_{\boldsymbol{k}}\right)\right] \\
t_{k+1} & \leftarrow \frac{1+\sqrt{1+4 t_{k}^{2}}}{2} \\
\boldsymbol{\eta}_{k+1} & \leftarrow \boldsymbol{\vartheta}_{k}^{S R}+\frac{t_{k}-1}{t_{k+1}}\left(\boldsymbol{\vartheta}_{k}^{S R}-\boldsymbol{\vartheta}_{k-1}^{S R}\right)
\end{aligned}
$$

where the positive soft-thresholding operator is applied coordinate-wise:

$$
S_{\tau, \alpha}^{+}[u]=\left\{\begin{array}{cl}
u-\frac{\tau}{2} & \text { if } \alpha+\frac{\tau}{2}>u>\frac{\tau}{2} \\
0 & \text { if } u \leq \frac{\tau}{2} \\
\alpha & \text { if } u \geq \alpha+\frac{\tau}{2}
\end{array}\right.
$$

and $\overline{\boldsymbol{H}}_{\Delta^{i}}^{*}$ represents the adjoint operator $\overline{\boldsymbol{H}}_{\Delta^{i}}$.

The soft-thresholding operator is simply generalized by considering a spatially varying threshold $\tau^{\prime}$. This is useful to account for the difference between the norms of each line of $\overline{\boldsymbol{H}}$. To avoid over-penalizing elements with a small norm, we define $\tau_{i}^{\prime}=\tau \sum_{j} w_{j} \overline{\boldsymbol{H}}_{i, j}^{2} / \sum_{j} w_{j}$. This normalization can be negligible in the field-of-view but is mandatory for out of the field reconstruction.

\section{EXPERIMENTS}

Compared to reconstructions based on a single low-resolution hologram, the MAP-SR is expected to recover higher frequency information in the hologram space, thereby leading to i) better resolved reconstructions, and ii) an enlargement of the reconstructed field-of-view. Demonstrating the two improvements requires different experimental configurations. To reconstruct an enlarged field-of-view (ii), a large recording distance is needed to capture the fringes coming from objects that are outside the field-of-view (due to pixel integration, the out-of-field signal is filtered out when the recording distance is too short). At the same time, large recording distances imply that high-frequency fringes from in-the-field objects fall outside the sensor area. The performance of the present method is demonstrated on both simulated and real holograms. The improvement in resolution (i) is demonstrated in the field-of-view of simulated holograms. The accuracy of shift estimation and the quality of the reconstruction are quantitatively compared with state-of-the-art approaches. The second expected improvement (ii) is shown on a stack of experimental holograms with a deliberately very low SNR in order to demonstrate that the proposed reconstruction is also efficient with noisy data. The quality of the reconstruction is compared with state-of-the-art reconstructions.

\section{A. Synthetic data}

Knowledge of the opacity distribution of the synthetic object and of the virtual relative shifts of the object with respect to the camera is used to quantify the errors in the reconstruction and in shift estimates.

\section{A.1. Simulated holograms}

In order to compute synthetic holograms, a plane transmittance object is simulated (Fig.5). The wave amplitude on the sensor is computed by propagating the amplitude wave of the transmittance plane to the sensor and considering a plane reference wave. The complex Fresnel free space propagation model is used. Finally, the intensity is calculated by the square modulus of the complex amplitude. This simulation, in contrast to the direct model used in the reconstruction (see section A), is closer to the optical model but is non-linear (which is why it was not used to derive the algorithm). It consists of computing a high-resolution complex amplitude of the propagated wave on the sensor and then computing its intensity (square modulus of the complex amplitude). The pixel integration effect is simulated by convolving the intensity by a 2D rectangle function of the final pixel size. To simulate a shifted hologram, the Fresnel kernel, which is 


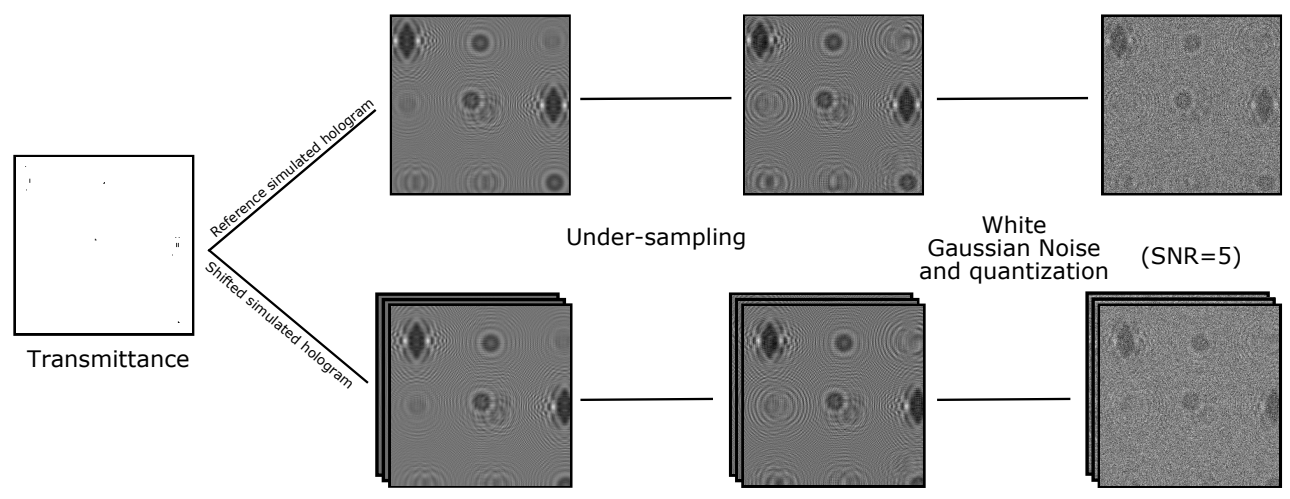

Fig. 4. Illustration of the holograms simulation pipeline to generate the stack.

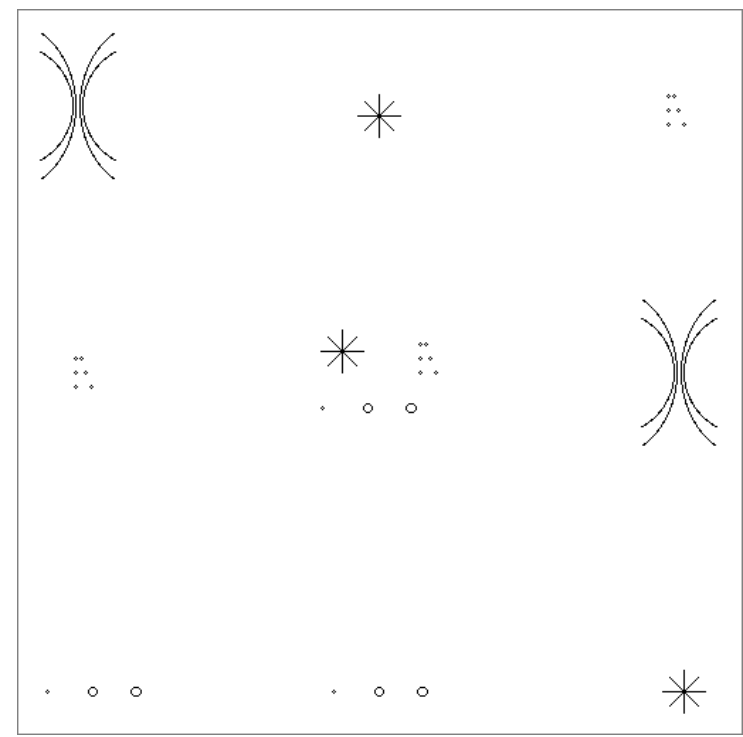

Fig. 5. Transmittance used to simulate holograms.

calculated from its analytical expression, is shifted with random shifts in $x$ and $y$ directions. To model a low resolution sensor, an under-sampling step by the same factor $f_{S R}$ is applied (see Fig. 4). Lastly, white Gaussian noise is added to simulate the electronic noise and a 12 bit quantization is applied on the holograms.

To simulate the hologram stacks, a $512 \times 512$ sampled transmittance distribution with a dot pitch of $3.7 \mu m$ is used (see Fig. 5). The wavelength is set to $662 \mathrm{~nm}$ and the object-sensor distance to $z=16.5 \mathrm{~mm}$. Holograms of $256 \times 256$ pixel with a pitch of $7.4 \mu \mathrm{m}$ and a fill-factor (i.e., active area over the total area of the pixel) of 0.5 are simulated. Stacks of $N=50$ holograms are generated with shifts uniformly distributed in the range $[-2,+2]$ pixels. The SNR (ratio of the magnitude of the signal to the standard deviation of the noise) is set to 5 in order to be close to an experimental value. The hyperparameter $\tau$ is set by minimizing the mean square deviation between the reconstruction of simulated holograms and the true transmittance.

\section{A.2. Accuracy of the hologram stack registration}

The first advantage of the MAP-SR method is a more accurate estimation of the shifts. In state-of-the-art approaches, a hologram is registered to another hologram [13, 23], whereas in the proposed approach, registration is between a noiseless model and the noisy data, as discussed in section A. Assuming a perfect reconstruction and ignoring boundary effects, a theoretical gain of $\sqrt{2}$ in precision for the estimated shifts is expected. In contrast to conventional methods which only consider the overlapping area between two holograms to perform registration, our approach exploits all the pixels of the holograms thus avoiding boundary effects. Nevertheless, incorrectly estimated opacities in our model may 


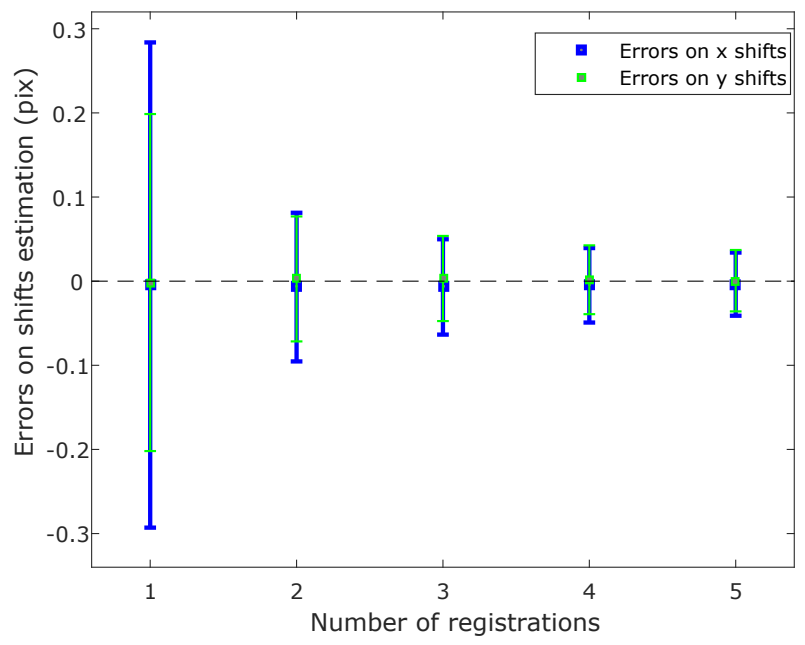

Fig. 6. Illustration of the refinement of $x$ and $y$ shift estimation throughout the opacity reconstruction process. The size of error bars represents 2 times the standard deviation on the shifts.

cause registration errors.

To check the improvement of the shift estimations in the reconstruction, simulations were run on 100 stacks of 50 holograms with the same random shifts and SNR as described in section A.1. The first shift guesses are obtained by correlation based registration of the $i^{\text {th }}$ hologram with the first hologram. The errors in the shifts are shown in figure 6 . They decrease during the course of the opacity reconstruction process. At the fifth iteration, the accuracy gain is 7.0 in $\mathrm{y}$ direction and 10.6 in $\mathrm{x}$ direction, which is a significant improvement in shift estimation.

\section{A.3. Quality of the reconstruction}

Four reconstructions were performed (Fig. 7-8): (a) back-propagation of a super-resolved hologram obtained with a state-of-the-art algorithm [6, 23], (b) MAP reconstruction using one image [17] and (c) MAP reconstruction using the same super-resolved hologram as in (a), (d) the proposed IP-MAP SR reconstruction.

Figure $7 \mathrm{a}$ shows that the back-propagation reconstruction suffers from twin image noise. Figure $7 \mathrm{~b}$ shows that the reconstruction using a single hologram is worse than the reconstructions using a stack of holograms displayed in figures $7 \mathrm{c}$ and $\mathrm{d}$. To better visualize the difference between figure $7 \mathrm{c}$ and figure $7 \mathrm{~d}$, zoomed versions are provided in figure 8. The SR MAP reconstruction (d) is the best reconstruction. For a quantitative comparison, the peak signal to noise ratio (PSNR) was computed for each reconstruction : (a) $16.2 \mathrm{~dB}$, (b) $24.1 \mathrm{~dB}$, (c) $28.0 \mathrm{~dB}$, (d) $39.2 \mathrm{~dB}$. It can be seen that the PSNR was improved by a factor of 2.4 from reconstruction (a) to (d).

\section{B. Experimental data}

Using IP-MAP SR reconstruction, the simulations showed an improvement in the reconstruction in the field-of-view. For experimental holograms, we demonstrate the improvement in the reconstruction outside the usual field-of-view using experimental holograms. To check the robustness of the proposed approach versus noise, the SNR of experimental holograms was deliberately reduced by spraying water on the light path. The setup is shown in figure 9. Sprayed water droplets create a realistic disturbance that decreases the SNR of the object signal. Between each of the $\mathrm{N}$ recordings, the object was randomly shifted transversely (versus $x$ and $y$ ). The test object is a metric crossed reticle (Edmund Optics) which has a sparse binary transmittance. The camera used for the experiments was a Prosilica GE 4900 camera. Its pixel pitch is $7.4 \mu \mathrm{m}$ and its fill-factor is around 1 . The recorded holograms are $1024 \times 1024$ pixels. The wavelength of the laser is $662 \mathrm{~nm}$. The camera was positioned at $z=283 \mathrm{~mm}$ from the object plane to record fringes of objects located outside the field-of-view.

Reconstruction was performed using a SR factor of 2 (the dot pitch of the opacity plane is $3.7 \mu \mathrm{m}$ ). The hyperparameter $\tau$ was set empirically. The width of the field-of-view increased by a factor of 3 leading to a $2.3 \mathrm{~cm}$ wide field-of-view and a surface of $5.2 \mathrm{~cm}^{2}$.

In figure 10, the same four reconstructions, as in the synthetic data case, are displayed for the purpose of comparison. It can be seen that the proposed reconstruction in figure 10d makes it possible to reconstruct an object 3 times wider than the sensor, whereas using the back-propagation reconstruction method, it is difficult to reconstruct beyond the 


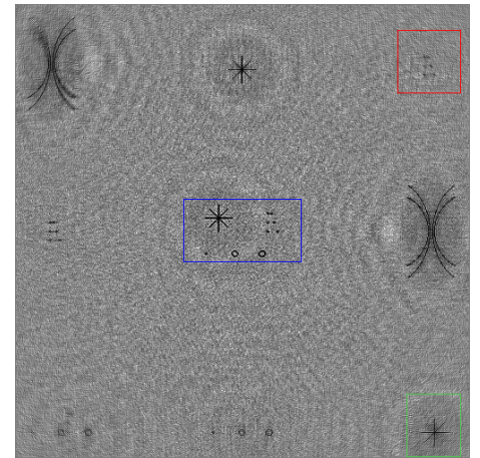

(a)

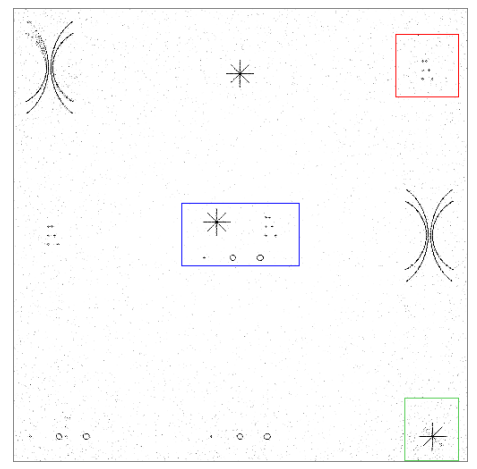

(c)

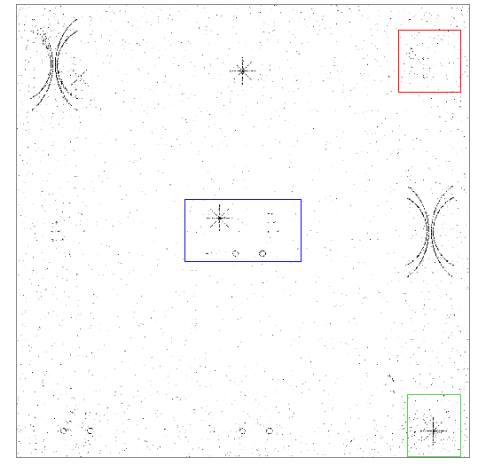

(b)

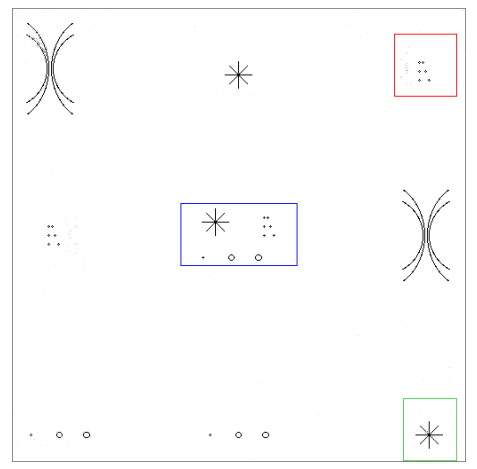

(d)

Fig. 7. Qualitative comparison of the reconstructions: (a) Back-propagation reconstruction using a super-resolved hologram [6, 13], (b) MAP reconstruction using 1 hologram [17], (c) MAP reconstruction using the same super-resolved hologram as in (a), (d) IP-MAP SR reconstruction using a stack of 50 holograms. The super-resolved hologram used for (a) and (c) is computed from the stack of 50 holograms with a state-of-the-art pixel super-resolution algorithm.

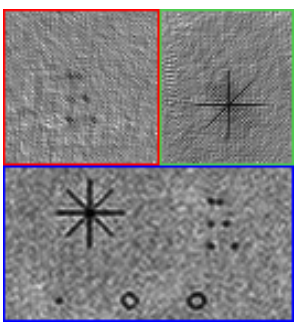

(a)

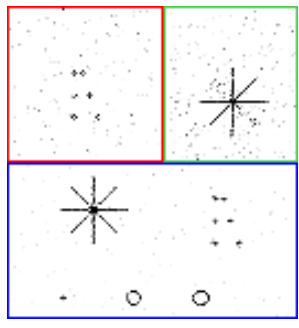

(c)

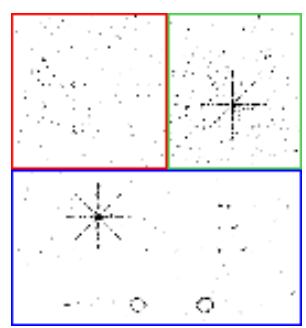

(b)

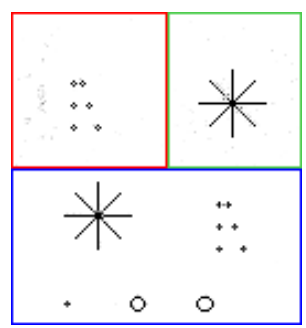

(d)

Fig. 8. Zoom on the reconstructions displayed on Figure 7. Colors indicate the zoomed areas. 


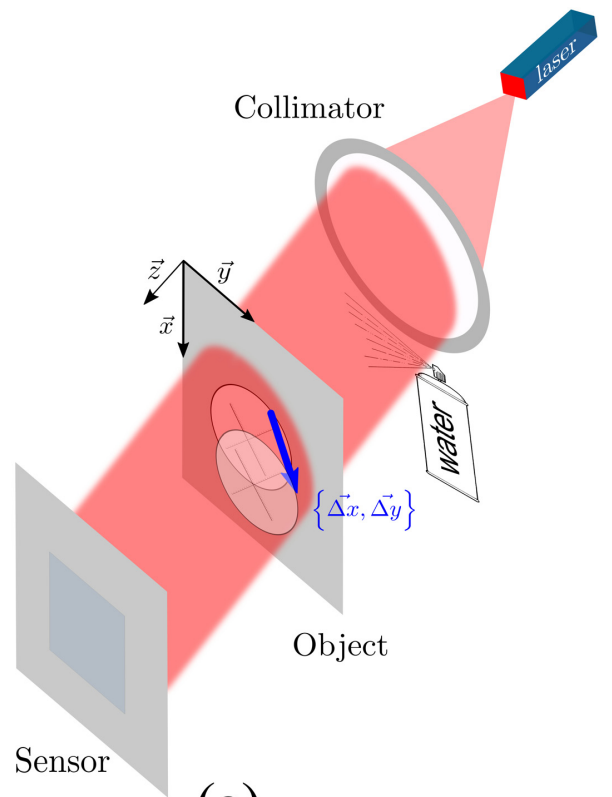

(a)

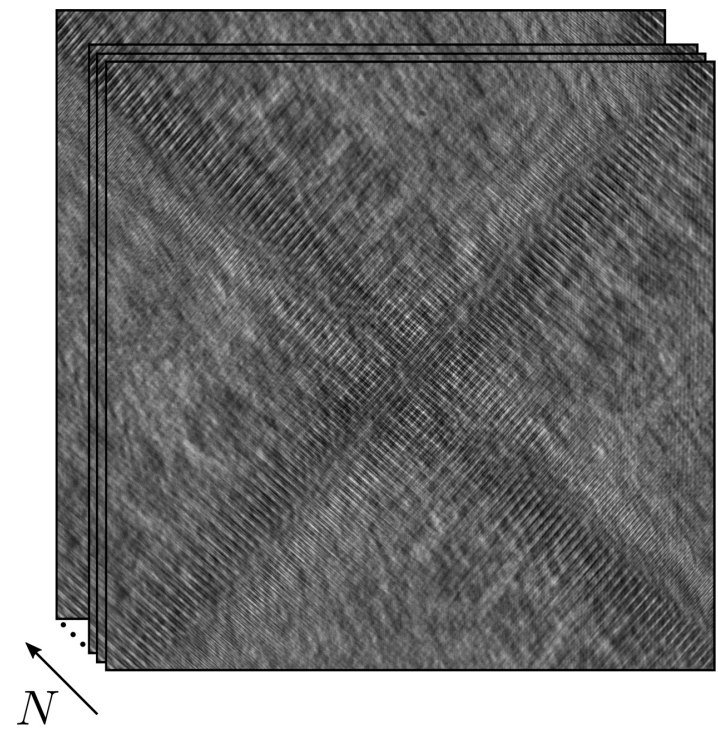

(b)

Fig. 9. Experimental holograms acquisition: (a) Experimental setup, (b) Illustration of a stack of captured holograms.

sensor field. This out-of-field reconstruction not only enables enlargement of the field-of-view but also renders shift estimation more accurate. Figure 11, shows that the in-field reconstruction is less noisy using MAP-SR reconstruction (d). The supplementary material (Visualization 1..4) makes it possible to zoom in to check the reconstruction of the border of the reticle. It should be noted that because the holograms are better registered with the MAP-SR approach (d), the hyperparameter is smaller in (d) than in (c) and hence easier to tune. In the example, the reconstruction (d) was obtained with a hyperparameter two times smaller than in (c). 


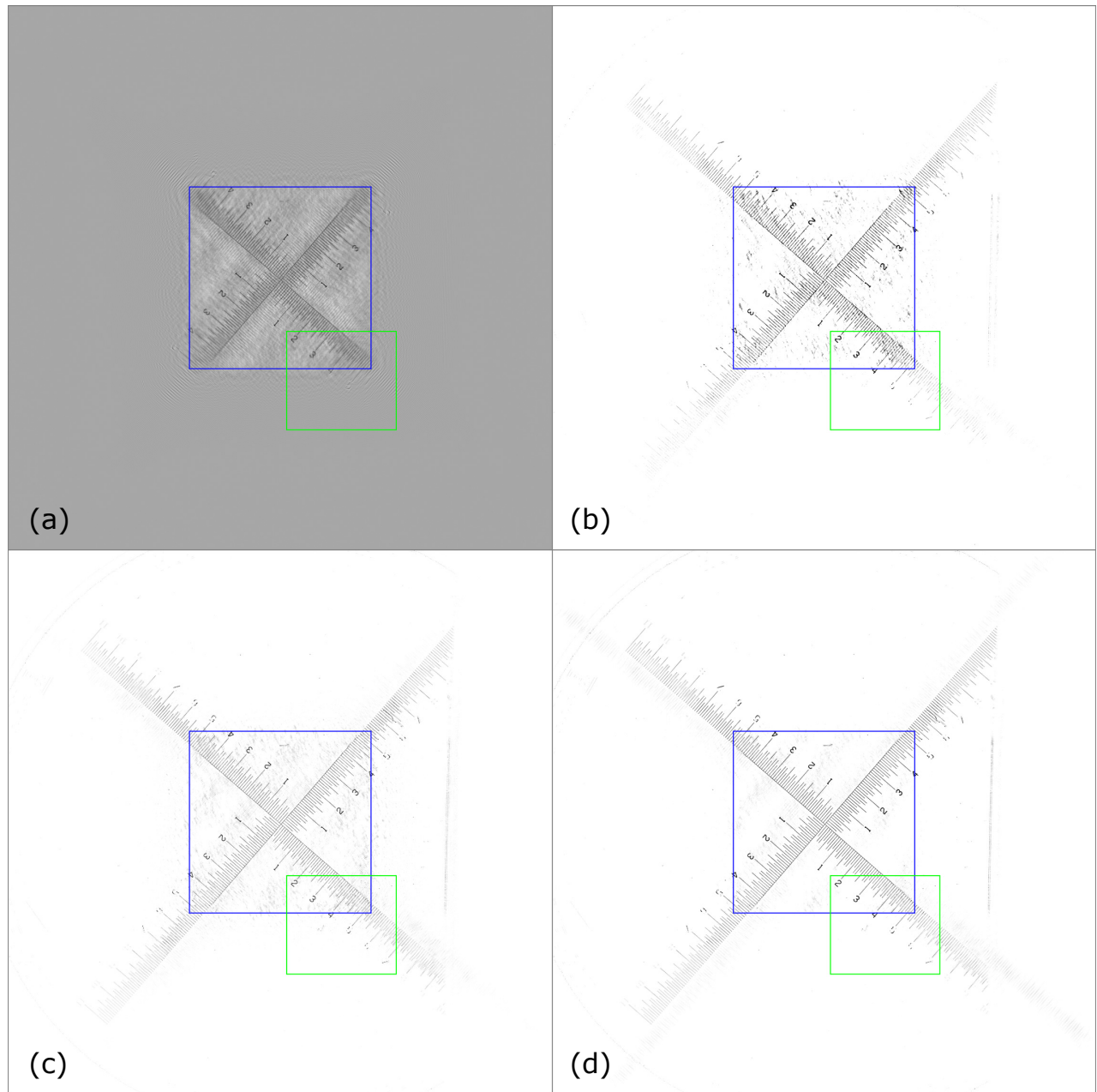

Fig. 10. Qualitative comparison of experimental hologram reconstructions: (a) Back-propagation reconstruction using a super-resolved hologram, (b) MAP reconstruction using 1 hologram [17], (c) MAP reconstruction using the same super-resolved hologram as in (a), (d) IP-MAP SR reconstruction using a stack of 15 holograms (see Visualization 1, 2, 3 and 4). The super-resolved hologram used for (a) and (c) was computed from the stack of 15 holograms with a stateof-the-art pixel super-resolution algorithm. The blue square in the center of the image represents the sensor borders. The green rectangle shows the zoomed areas displayed in figure 11.

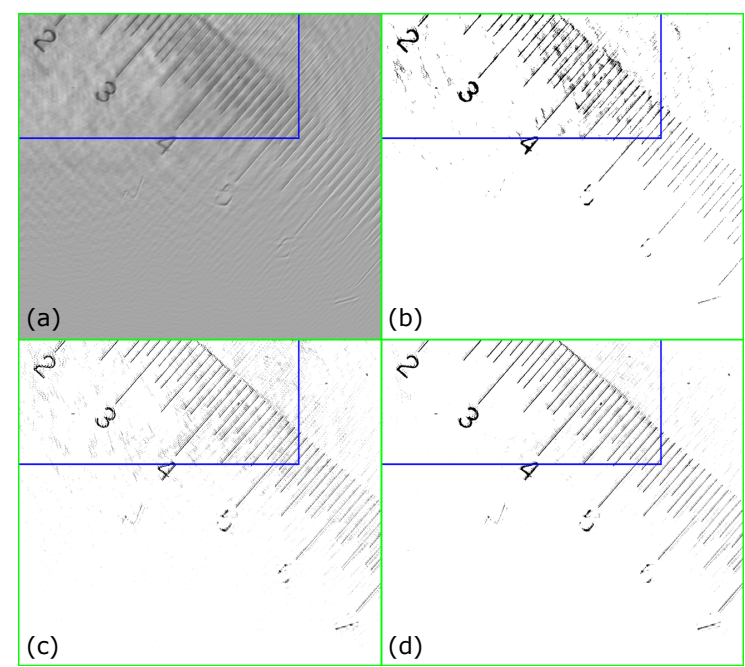

Fig. 11. Zoom on the reconstructions displayed on Figure.10.a-d 


\section{CONCLUSION}

An inverse problem approach was successfully applied to the super-resolved reconstruction of holograms. Starting from a collection of unregistered holograms, the proposed method alternates between a step of sub-pixel registration of the holograms and a step of joint inversion of all holograms. This method differs from recent super-resolution methods applied in digital holography and lensless imaging in two major aspects: (i) sub-pixel hologram registration is performed by matching holograms with a (noiseless) model obtained from the previous reconstruction, rather than by matching holograms to a reference (noisy) hologram; (ii) the reconstruction method inverts the hologram formation model by including prior knowledge (bound constraints, sparsity), rather than by light back-propagation. Those two modifications result in better reconstructions with reduced artefacts (twin-images, border effects), an extended field-ofview, a better signal-to-noise ratio, and improved spatial resolution. These improvements come at the cost of increased computational complexity compared to existing pixel super-resolution approaches. We believe the improvement in the reconstructions outweighs the drawback of this higher computational cost. In applications where reconstruction time must be kept short, special effort should be invested in accelerating the Fresnel propagator, using GPU implementations, and refining the minimization strategy, for example using continuation schemes.

\section{Fundings}

This project is funded by the Agence Nationale de la Recherche (ANR) (ANR-1 1-IDEX-0007, ANR-11-LABX-0063); DETECTION-CNRS DEFI IMAGIn 2015.

Funding for this project was provided by a grant from la Région Auvergne-Rhône-Alpes.

\section{REFERENCES}

1. D. Gabor et al., "A new microscopic principle," Nature 161, 777-778 (1948).

2. H. Royer, "Holographic velocimetry of submicron particles," Optics Communications 20, 73-75 (1977).

3. U. Schnars and W. P. Jüptner, "Digital recording and reconstruction of holograms in hologram interferometry and shearography," Applied optics 33, 43734377 (1994).

4. L. Repetto, E. Piano, and C. Pontiggia, "Lensless digital holographic microscope with light-emitting diode illumination," Optics letters 29, 1132-1134 (2004).

5. U. Schnars and W. P. Jüptner, "Digital recording and numerical reconstruction of holograms," Measurement science and technology 13, R85 (2002).

6. T. M. Kreis, "Handbook of Holographic Interferometry, Optical and Digital Methods," Wiley-VCH (2005).

7. N. Verrier and M. Atlan, "Off-axis digital hologram reconstruction: some practical considerations," Applied optics 50, H136-H146 (2011).

8. D. Tseng, O. Mudanyali, C. Oztoprak, S. O. Isikman, I. Sencan, O. Yaglidere, and A. Ozcan, "Lensfree microscopy on a cellphone," Lab on a Chip 10, 1787-1792 (2010).

9. C. Allier, G. Hiernard, V. Poher, and J. Dinten, "Bacteria detection with thin wetting film lensless imaging," Biomedical optics express 1, 762-770 (2010).

10. O. Mudanyali, E. McLeod, W. Luo, A. Greenbaum, A. F. Coskun, Y. Hennequin, C. P. Allier, and A. Ozcan, "Wide-field optical detection of nanoparticles using on-chip microscopy and self-assembled nanolenses," Nature photonics 7, 247-254 (2013).

11. S. Seo, T.-W. Su, D. K. Tseng, A. Erlinger, and A. Ozcan, "Lensfree holographic imaging for on-chip cytometry and diagnostics," Lab on a Chip 9, 777-787 (2009).

12. C. Wang, X. Zhong, D. B. Ruffner, A. Stutt, L. A. Philips, M. D. Ward, and D. G. Grier, "Holographic characterization of protein aggregates," Journal of pharmaceutical sciences 105, 1074-1085 (2016).

13. W. Bishara, T.-W. Su, A. F. Coskun, and A. Ozcan, "Lensfree on-chip microscopy over a wide field-of-view using pixel super-resolution," Optics express 18, 11181-11191 (2010).

14. S. Sotthivirat and J. A. Fessler, "Penalized-likelihood image reconstruction for digital holography," JOSA A 21, 737-750 (2004).

15. F. Soulez, L. Denis, C. Fournier, É. Thiébaut, and C. Goepfert, "Inverse-problem approach for particle digital holography: accurate location based on local optimization," JOSA A 24, 1164-1171 (2007).

16. F. Soulez, L. Denis, E. Thiébaut, C. Fournier, and C. Goepfert, "Inverse problem approach in particle digital holography: out-of-field particle detection made possible," JOSA A 24, 3708-3716 (2007).

17. L. Denis, D. Lorenz, E. Thiébaut, C. Fournier, and D. Trede, "Inline hologram reconstruction with sparsity constraints," Optics letters 34, 3475-3477 (2009).

18. A. Bourquard, N. Pavillon, E. Bostan, C. Depeursinge, and M. Unser, "A practical inverse-problem approach to digital holographic reconstruction," Optics express 21, 3417-3433 (2013).

19. S. Lim, D. L. Marks, and D. J. Brady, "Sampling and processing for compressive holography [invited]," Applied Optics 50, H75-H86 (2011).

20. D. J. Brady, K. Choi, D. L. Marks, R. Horisaki, and S. Lim, "Compressive holography," Optics express 17, 13040-13049 (2009).

21. Y. Rivenson, A. Stern, and B. Javidi, "Compressive fresnel holography," Journal of Display Technology 6, 506-509 (2010).

22. S. C. Park, M. K. Park, and M. G. Kang, "Super-resolution image reconstruction: a technical overview," IEEE signal processing magazine 20, 21-36 (2003).

23. P. Vandewalle, S. Süsstrunk, and M. Vetterli, "A Frequency Domain Approach to Registration of Aliased Images with Application to Super-Resolution," EURASIP Journal on Applied Signal Processing (special issue on Super-resolution) 2006, Article ID 71459, 14 pages (2006).

24. N. Verrier and C. Fournier, "Digital holography super-resolution for accurate three-dimensional reconstruction of particle holograms," Optics letters $\mathbf{4 0}$, 217-220 (2015).

25. N. Verrier, C. Fournier, and T. Fournel, "3d tracking the brownian motion of colloidal particles using digital holographic microscopy and joint reconstruction," Applied Optics 54, 4996-5002 (2015).

26. M. Marim, E. Angelini, J.-C. Olivo-Marin, and M. Atlan, "Off-axis compressed holographic microscopy in low-light conditions," Optics letters 36, 79-81 (2011). 
27. M. M. Marim, M. Atlan, E. Angelini, and J.-C. Olivo-Marin, "Compressed sensing with off-axis frequency-shifting holography," Optics letters 35, 871-873 (2010).

28. W. G. Joseph, "Introduction to fourier optics," McGraw Hill 10, 160-165 (1996).

29. T. M. Kreis, "Frequency analysis of digital holography with reconstruction by convolution," Optical Engineering 41, 1829-1839 (2002).

30. L. Denis, D. Lorenz, E. Thiébaut, C. Fournier, and D. Trede, "Inline hologram reconstruction with sparsity constraints," Optics letters 34, 3475-3477 (2009).

31. C. Fournier, L. Denis, M. Seifi, and T. Fournel, "Digital hologram processing in on-axis holography," Multi-dimensional Imaging 0, 51-73 (2014).

32. R. C. Hardie, K. J. Barnard, and E. E. Armstrong, "Joint map registration and high-resolution image estimation using a sequence of undersampled images," Image Processing, IEEE Transactions on 6, 1621-1633 (1997).

33. S. Farsiu, D. Robinson, M. Elad, and P. Milanfar, "Advances and challenges in super-resolution," International Journal of Imaging Systems and Technology 14, 47-57 (2004).

34. E. Thiébaut, "Optimization issues in blind deconvolution algorithms," Astronomical Telescopes and Instrumentation pp. 174-183 (2002).

35. A. Beck and M. Teboulle, "A fast iterative shrinkage-thresholding algorithm for linear inverse problems," SIAM journal on imaging sciences 2, 183-202 (2009).

36. Y. Endo, T. Shimobaba, T. Kakue, and T. Ito, "GPU-accelerated compressive holography," Optics Express 24, 8437 (2016). 